\section{Bendamustin: Neuer Handelsname nach EU-Zulassung}

Für Bendamustin, das in Deutschland unter dem Handelsnamen Ribomustin ${ }^{\circledast}$ (Mundipharma GmbH, Limburg) seit Jahren erfolgreich zur Behandlung bei malignen Lymphomen und multiplem Myelom eingesetzt wird, ist eine europäische Zulassung erteilt worden. Seit dem 15. Dezember 2011 ist Bendamustin daher in Europa und auch in Deutschland unter dem Warennamen Levact ${ }^{\circledast}$ erhältlich. Die kostenlose medizinische Mundipharma Info-Line ist unter 0800 / 8551111 erreichbar.

\title{
Maligner Aszites
}

\section{Verkürzte Infusionsdauer von Catumaxomab zugelassen}

Die Europäische Kommission hat Catumaxomabjetzt auch mit einer auf drei Stunden verkürzten Infusionsdauer zur Behandlung des malignen Aszites zugelassen. Catumaxomab wurde erstmals im April 2009 zur intraperitonealen Behandlung des malignen Aszites bei Patienten mit EpCAM-positivem Karzinom zugelassen.

Die Basis der Zulassungserweiterung war eine Sicherheitsanalyse der Daten von sieben Studien zur Anwendung von Catumaxomab (Removab ${ }^{\oplus}$ ) als 3-Stunden-Infusion (Finas D et al., 2011, EMCC:\#8032, poster presentation). In dieser Analyse wurden die Häu- figkeit und die Schwere der Nebenwirkungen bei dreistündiger Infusion mit denen bei sechsstündiger Infusion von Catumaxomab verglichen. Es zeigte sich, dass das Nebenwirkungsspektrum vergleichbar war.

Der trifunktionale Antikörper Catumaxomab bindet gleichzeitig an Tumorzellen, TZellen und akzessorische Zellen des Immunsystems und induziert damit eine Immunreaktion gegen Tumorzellen. Die immunologische Zerstörung von peritonealen Tumorzellen führt zur nachhaltigen Reduktion der Aszitesbildung (Fachinformation Removab $^{\circledast}$, Stand September 2011).

Quelle: Information der Fresenius Biotech GmbH

\section{Misteltherapie}

\section{Ergänzung und Unterstützung zu konventionellen Tumortherapien}

Die anthroposophische Misteltherapie kann etablierte onkologische Standardtherapien im Sinne einer umfassenderen und ganzheitlichen Versorgung ergänzen.

Mistelextrakte, wie z.B. der anthroposophische Mistelextrakt Iscador ${ }^{\circledast}$, sind komplexe Vielstoffgemische. Die antikarzinogenen Eigenschaften der biologisch aktiven Inhaltsstoffe, vor allem der Mistellektine und Viscotoxine (Tab.), wurden experimentell nachgewiesen. Sie prädestinieren die als Halbparasit auf verschiedenen Wirtsbäumen lebende weißbeerige Mistel (Viscum album L.) für den Einsatz in der Tumortherapie. „Mistel in Form von Tee oder Tropfen ist allerdings unwirksam, denn bei der Herstellung werden die potenten Wirkstoffe zerstört", so Dr. Frank Meyer, Nürnberg. In seiner ganzheitlich orientierten Hausarztpraxis wendet Meyer Mistelextrakt-Injektionen erfolgreich an nicht als „alternative Therapie“, sondern als Ergänzung der Tumortherapie, wie Meyer betonte. „Während die konventionellen Standardtherapien und Nachsorgeuntersuchungen meist in spezialisierten Krebszentren durchgeführt werden, wenden sich Tu-

Inhaltsstoffe - anthroposophisches Mistelextrakt

$\begin{array}{ll}\begin{array}{l}\text { Stoffklasse } \\ \text { Mistellektine I, II, III }\end{array} & \begin{array}{l}\text { Wirkung auf die Tumorzellen } \\ \text { Zytotoxizität durch } \\ \text { Hemmung der ribosomalen } \\ \text { Proteinsynthese } \\ \text { Apoptose-Induktion } \\ \text { geringe Zytotoxizität }\end{array} \\ \begin{array}{l}\text { Chitin-bindendes } \\ \text { Mistellektin }\end{array} & \begin{array}{l}\text { Zytotoxizität durch } \\ \text { Vembranolyse } \\ \text { Vemmung der DNA-, RNA- } \\ \text { Hemine }\end{array} \\ & \begin{array}{l}\text { und Proteinsynthese } \\ \end{array}\end{array}$

nach Iscador ${ }^{\oplus}$, Wissenschaftliche Informationen, Weleda AG, 2010 morpatienten in allen anderen Fragen meist an ihren Hausarzt." Dazu zählen nach seiner Erfahrung häufig unspezifische Beschwerden, wie z.B. Erschöpfung, Ängste, Appetitlosigkeit oder Wundheilungsstörungen. Dagegen können Mistelextrakte eingesetzt werden, da sie die körpereigene Abwehr aktivieren, Appetit und Lebensfreude steigern sowie Nebenwirkungen von Chemo- oder Strahlentherapie verringern können.

\section{Selbstheilungskräfte der Patienten werden angeregt}

Dr. Christian Büttner, Kassel, hat die Erfahrung gemacht, dass Patienten schon wenige Tage nach Anwendung von Mistelextrakt eine Erleichterung ihrer Situation verspüren. Daher eignet sich die Therapie Büttner zufolge gut als "Türöffner" und "Wegweiser" für den Patienten. Ganz im Sinne der integrativen Medizin, die nicht nur auf die Krankheit zielt, sondern auch die Selbstheilungskräfte des Organismus anregt. „Der Patient übernimmt eine aktive Rolle - sowohl bei der Überwindung der Krankheit als auch beim Erhalt der Gesundheit. Zudem ist die Durchführung der Therapie relativ einfach in fast allen Fällen können die Patienten nach kurzer Einweisung die Injektionen selbst vornehmen", ergänzte Büttner. $C B$

Quelle: Veranstaltung der Weleda AG 